acute pulmonary embolism: a prospective validation study. Circulation 2011; 124: 2716-2724.

3 Aujesky D, Roy PM, Verschuren F, et al. Outpatient versus inpatient treatment for patients with acute pulmonary embolism: an international, open-label, randomised, non-inferiority trial. Lancet 2011; 378: 41-48.

DOI: $10.1183 / 09031936.00124612$

\title{
Re-think first-line tuberculosis treatment
}

\section{To the Editor:}

In the June issue of the European Respiratory Journal (ERJ), A. Skrahina and co-workers reported alarming rates of multidrug-resistant tuberculosis (MDR-TB) in Minsk, Belarus. In their survey, they diagnosed MDR-TB in 35.3\% of new patients and $76.5 \%$ of previously treated patients [1]. The reflex response to such figures is to strengthen classical infection control activities, to intensify case detection, and to strengthen adherence to and rational use of the first-line antituberculosis regimen. Unfortunately, with the MDR-TB rates now emerging from Eastern Europe and Central Asia, it is questionable how appropriate the current first-line regimen and its rigid use in newly diagnosed cases are in that setting. Is it not time to consider an entirely new first-line treatment for countries or regions where, for example, $>25 \%$ of tuberculosis in previously untreated patients is MDR-TB? In a different setting, that of community-acquired pneumonia, the use of macrolides as first-line therapy despite $25 \%$ macrolide resistance among its causative agents results in therapy-attributable mortality in around one in 100 persons treated [2]. Generally speaking, should we not consider revising the current first-line regimen once a yet-to-be-determined level of MDR-TB is measured, in order to prevent therapy-attributable mortality?

Prolonged continuation of current first-line regimens in highprevalence MDR-TB settings favours the further emergence of such strains. Strains with resistance to the initial treatment choice are given a selective advantage and opportunity to spread in the community or nosocomially, which they would not otherwise have. Initial treatment with an ineffective regimen also provides conditions which may favour their adaptation towards preservation of transmissibility (e.g. by acquiring so-called compensatory mutations) [3].

Recent studies have found that regimens including bedaquiline (TMC207), PA-824, pyrazinamide, moxifloxacin and clofazimine have similar or stronger bactericidal activity than the current regimen of isoniazid, rifampicin and pyrazinamide with ethambutol or streptomycin. These novel compounds lack any cross-resistance with existing drugs [4, 5]. Different strategies for their implementation can be used. First, these regimens could serve as a universal regimen for settings where MDR-TB is so prevalent that it is unsafe and perhaps unethical to continue by just enforcing the old first-line regimen.

As a second strategy would entail the use of a new universal regimen followed by de-escalation to the current first-line regimen for patients infected with strains treatable with current first-line therapy. Unrestricted access to rapid molecular resistance detection or phenotypic susceptibility testing is critical to this strategy. Such screening also provides the opportunity for separate quarantine measures for patients with sensitive tuberculosis, who rapidly become uninfectious after initiation of therapy. Where possible, these patients would not be admitted to hospitals where they are at risk of superinfection with MDR-TB. This may help to slow the truly alarming increases in resistance rates seen in areas where prolonged in-patient treatment of tuberculosis is the norm.

Of course, the switch to a universal regimen can only be successful if it is part of a stable programme that also involves rigid infection control activities, active case detection, close monitoring of individual treatment adherence and rational use of the novel drugs, as set out in the recent review series in the $E R J$, introduced and summarised by ZuMLA et al. [6].

The use of an initial regimen that is ineffective for a sizeable proportion of the patients and encourages the spread and further adaptation of resistant strains is unacceptable. The tuberculosis community must now push forward and start clinical trials of universal regimens that are equally active against pan-susceptible and MDR-TB.

\section{Jakko van Ingen* and Richard M. Anthony ${ }^{\#}$}

*Dept of Medical Microbiology, Radboud University Nijmegen Medical Centre, Nijmegen, and "KIT Biomedical Research, Royal Tropical Institute, Amsterdam, The Netherlands.

Correspondence: J. van Ingen, Dept of Medical Microbiology (574), Radboud University Medical Centre, PO Box 9101, 6500HB Nijmegen, The Netherlands. E-mail: vaningen.jakko@gmail.com

Statement of Interest: None declared.

\section{REFERENCES}

1 Skrahina A, Hurevich H, Zalutskaya A, et al. Alarming levels of drug-resistant tuberculosis in Belarus: results of a survey in Minsk. Eur Respir J 2012; 39: 1425-1431.

2 Daneman N, Low DE, McGeer A, et al. At the threshold: defining clinically meaningful resistance thresholds for antibiotic choice in community-acquired pneumonia. Clin Infect Dis 2008; 46: 1131-1138.

3 Comas I, Borrell S, Roetzer A, et al. Whole-genome sequencing of rifampicin-resistant Mycobacterium tuberculosis strains identifies compensatory mutations in RNA polymerase genes. Nat Genet 2011; 44: 106-110.

4 Williams K, Minkowski A, Amoabeng O, et al. Sterilizing activities of novel combinations lacking first- and second-line drugs in a murine model of tuberculosis. Antimicrob Agents Chemother 2012; 56 : 3114-3120. 
5 Diacon AH, Dawson R, von Groote-Bidlingmaier F, et al. 14-day bactericidal activity of PA-824, bedaquiline, pyrazinamide, and moxifloxacin combinations: a randomised trial. Lancet 2012; 380: 986-993.
6 Zumla A, Blasi F, Raviglione M. Rational use of anti-tuberculosis drugs in the EU: better patient care and less drug resistance. Eur Respir J 2012; 39: 802-804.

DOI: 10.1183/09031936.00112612

\section{Adjusting diffusing capacity of the lung for carbon monoxide for haemoglobin level}

\section{To the Editor:}

The joint statements on lung function testing by the American Thoracic Society (ATS)/European Respiratory Society (ERS) Task Force provided useful recommendations for standardisation of the tests in daily practice [1]. However, the equations for adjusting diffusing capacity of the lung for carbon monoxide $(\mathrm{DL}, \mathrm{CO})$ for the haemoglobin $(\mathrm{Hb})$ level (equations 13 and 14 in the original document) may be confusing [1]. In daily clinical practice, instead of changing the predicted reference value, we tend to adjust the observed $D L, C O$ value as if the patient had a normal $\mathrm{Hb}$ level [2-4]. Therefore, as suggested by most guidelines [2, 4], we suggest new equations for adjustment, as shown in table 1 .

It is well known that anaemia decreases observed $D L, C O$ by decreasing the area for diffusion due to a reduction in pulmonary capillary haemoglobin [1-4]. For example, in our suggested equation for adult males and adolescents, a $\mathrm{Hb}$ level $<14.6 \mathrm{~g} \cdot \mathrm{dL}^{-1}$ makes $(10.22+\mathrm{Hb}) /(1.7 \times \mathrm{Hb})>1$, making $D \mathrm{~L}, \mathrm{CO}_{\text {adjusted for } \mathrm{Hb}}>\mathrm{DL}, \mathrm{CO}_{\text {observed }}$ to eliminate the effect of anaemia. This adjustment makes DL,CO more standardised for assessing pulmonary conditions.

As the joints statements by the ATS/ERS Task Force are widely used as a guide for standardisation of clinical practice, we believe that clarifying this point is important.

\section{Ming-Ju Tsai*,\#, ${ }^{*},+$, Chih-Jen Yang ${ }^{*},+,+$, , Jhi-Jhu Hwang ${ }^{*, f}$, and Ming-Shyan Huang ${ }^{*,+,}$}

*Division of Pulmonary and Critical Care Medicine, Dept of Internal Medicine, Kaohsiung Medical University Hospital, Kaohsiung Medical University, "Dept of Internal Medicine, Kaohsiung Municipal HsiaoKang Hospital, Kaohsiung
Medical University, "Dept of Internal Medicine, Kaohsiung Municipal TaTung Hospital, Kaohsiung Medical University, ${ }^{+}$Graduate Institute of Medicine, School of Medicine, College of Medicine, Kaohsiung Medical University, ${ }^{\S}$ Dept of Internal Medicine, School of Medicine, College of Medicine, Kaohsiung Medical University, and ${ }^{f}$ Dept of Respiratory Therapy, College of Medicine, Kaohsiung Medical University, Kaohsiung, Taiwan.

Correspondence: M-S. Huang, Division of Pulmonary and Critical Care Medicine, Dept of Internal Medicine, Kaohsiung Medical University Hospital, Kaohsiung Medical University, No. 100 Tzyou 1st Road, Kaohsiung 807, Taiwan. E-mail: shyang@cc.kmu.edu.tw

Statement of Interest: None declared.

\section{REFERENCES}

1 Macintyre N, Crapo RO, Viegi G, et al. Standardisation of the singlebreath determination of carbon monoxide uptake in the lung. Eur Respir J 2005; 26: 720-735.

2 American Thoracic Society. Single-breath carbon monoxide diffusing capacity (transfer factor). Recommendations for a standard technique - 1995 update. Am J Respir Crit Care Med 1995; 152: 2185-2198.

3 Marrades RM, Diaz O, Roca J, et al. Adjustment of DL,CO for hemoglobin concentration. Am J Respir Crit Care Med 1997; 155: 236-241.

4 American Association for Respiratory Care. AARC clinical practice guideline: single-breath carbon monoxide diffusing capacity, 1999 update. Respir Care 1999; 44: 539-546.

DOI: 10.1183/09031936.00152212

TABLE 1 Summary of the suggested changes

Original equations (to adjust predicted value) [1]

$D \mathrm{~L}, \mathrm{CO}_{\text {predicted for } \mathrm{Hb}}=D \mathrm{~L}, \mathrm{CO}_{\text {predicted }} \times(1.7 \times \mathrm{Hb}) /(10.22+\mathrm{Hb})$

$\mathrm{DL}, \mathrm{CO}_{\text {predicted for } \mathrm{Hb}}=\mathrm{DL}, \mathrm{CO}_{\text {predicted }} \times(1.7 \times \mathrm{Hb}) /(9.38+\mathrm{Hb})$
Suggested equations (to adjust observed value)

$D \mathrm{~L}, \mathrm{CO}_{\text {adjusted for } \mathrm{HL}}=D \mathrm{~L}, \mathrm{CO}_{\text {observed }} \times(10.22+\mathrm{Hb}) /(1.7 \times \mathrm{Hb})$

$D \mathrm{~L}, \mathrm{CO}_{\text {adjusted for } \mathrm{Hb}}=\mathrm{DL}, \mathrm{CO}_{\text {observed }} \times(9.38+\mathrm{Hb}) /(1.7 \times \mathrm{Hb})$
For adult males and adolescents For adult females and children $<15$ yrs of age

DL,CO: diffusing capacity of the lung for carbon monoxide; $\mathrm{Hb}$ : haemoglobin. 